\title{
THE DISPLACED DUSTY INTERSTELLAR MEDIUM OF NGC 3077: TIDAL STRIPPING IN THE M 81 TRIPLET
}

\author{
F. Walter ${ }^{1}$, K. Sandstrom ${ }^{1}$, G. Aniano ${ }^{2}$, D. Calzetti $^{3}$, K. Croxall ${ }^{4}$, D. A. Dale ${ }^{5}$, B. T. Draine ${ }^{2}$ C. $_{\text {Engelbracht }}^{6}$, \\ J. Hinz ${ }^{6}$, R. C. Kennicutt ${ }^{7}$, M. Wolfire ${ }^{8}$, L. Armus ${ }^{9}$, P. Beirão ${ }^{9}$, A. D. Bolatto ${ }^{8}$, B. Brandi ${ }^{10}$, A. Crocker $^{3}$, \\ M. Galametz ${ }^{7}$, B. Groves ${ }^{10}$, C.-N. HaO ${ }^{11}$, G. Helou ${ }^{12}$, L. Hunt ${ }^{13}$, J. KodA ${ }^{14}$, O. Krause ${ }^{1}$, A. Leroy ${ }^{15,20}$, S. Meidt ${ }^{1}$, \\ E. J. Murphy ${ }^{9}$, N. Rahman ${ }^{8}$, H.-W. Rix ${ }^{1}$, H. Roussel ${ }^{16}$, M. Sauvage ${ }^{17}$, E. Schinnerer ${ }^{1}$, R. Skibba ${ }^{6}$, J. D. Smith ${ }^{4}$, \\ C. D. Wilson ${ }^{18}$, AND S. ZibetTi ${ }^{19}$ \\ ${ }^{1}$ Max-Planck-Institut für Astronomie, Königstuhl 17, D-69117 Heidelberg, Germany; walter@mpia.de, sandstrom@mpia.de \\ ${ }^{2}$ Department of Astrophysical Sciences, Princeton University, Princeton, NJ 08544, USA \\ ${ }^{3}$ Department of Astronomy, University of Massachusetts, Amherst, MA 01003, USA \\ ${ }^{4}$ Department of Physics and Astronomy, University of Toledo, Toledo, OH 43606, USA \\ ${ }^{5}$ Department of Physics \& Astronomy, University of Wyoming, Laramie, WY 82071, USA \\ ${ }^{6}$ Steward Observatory, University of Arizona, Tucson, AZ 85721, USA \\ ${ }^{7}$ Institute of Astronomy, University of Cambridge, Madingley Road, Cambridge CB3 0HA, UK \\ ${ }^{8}$ Department of Astronomy, University of Maryland, College Park, MD 20742, USA \\ ${ }^{9}$ Spitzer Science Center, California Institute of Technology, MC 314-6, Pasadena, CA 91125, USA \\ ${ }^{10}$ Leiden Observatory, Leiden University, P.O. Box 9513, 2300 RA Leiden, The Netherlands \\ ${ }^{11}$ Tianjin Astrophysics Center, Tianjin Normal University, Tianjin 300387, China \\ ${ }^{12}$ NASA Herschel Science Center, IPAC, California Institute of Technology, Pasadena, CA 91125, USA \\ ${ }^{13}$ INAF-Osservatorio Astrofisico di Arcetri, Largo E. Fermi 5, 50125 Firenze, Italy \\ ${ }^{14}$ Department of Physics and Astronomy, SUNY Stony Brook, Stony Brook, NY 11794-3800, USA \\ ${ }^{15}$ National Radio Astronomy Observatory, 520 Edgemont Road, Charlottesville, VA 22903, USA \\ ${ }^{16}$ Institut d'Astrophysique de Paris, UMR7095 CNRS, Université Pierre \& Marie Curie, 98 bis Boulevard Arago, 75014 Paris, France \\ ${ }^{17}$ CEA/DSM/DAPNIA/Service d'Astrophysique, UMR AIM, CE Saclay, 91191 Gif sur Yvette Cedex, France \\ ${ }^{18}$ Department of Physics \& Astronomy, McMaster University, Hamilton, Ontario L8S 4M1, Canada \\ ${ }^{19}$ Dark Cosmology Centre, Niels Bohr Institute, University of Copenhagen, Juliane Maries Vej 30, DK-2100 Copenhagen, Denmark \\ Received 2010 November 5; accepted 2010 November 29; published 2010 December 13
}

\begin{abstract}
We present the detection of extended $\left(\sim 30 \mathrm{kpc}^{2}\right)$ dust emission in the tidal $\mathrm{H}$ i arm near NGC 3077 (member of the M 81 triplet) using SPIRE on board Herschel. Dust emission in the tidal arm is typically detected where the H I column densities are $>10^{21} \mathrm{~cm}^{-2}$. The SPIRE band ratios show that the dust in the tidal arm is significantly colder $(\sim 13 \mathrm{~K})$ than in NGC 3077 itself $(\sim 31 \mathrm{~K})$, consistent with the lower radiation field in the tidal arm. The total dust mass in the tidal arm is $\sim 1.8 \times 10^{6} M_{\odot}$ (assuming $\beta=2$ ), i.e., substantially larger than the dust mass associated with NGC $3077\left(\sim 2 \times 10^{5} M_{\odot}\right)$. Where dust is detected, the dust-to-gas ratio is $(6 \pm 3) \times 10^{-3}$, consistent within the uncertainties with what is found in NGC 3077 and nearby spiral galaxies with Galactic metallicities. The faint $\mathrm{H}$ II regions in the tidal arm cannot be responsible for the detected enriched material and are not the main source of the dust heating in the tidal arm. We conclude that the interstellar medium (atomic $\mathrm{HI}$, molecules, and dust) in this tidal feature was pre-enriched and stripped off NGC 3077 during its recent interaction $\left(\sim 3 \times 10^{8} \mathrm{yr}\right.$ ago $)$ with M 82 and M 81. This implies that interaction can efficiently remove heavy elements and enriched material (dust and molecular gas) from galaxies. As interactions were more frequent at large look-back times, it is conceivable that they could substantially contribute (along with galactic outflows) to the enrichment of the intergalactic medium.
\end{abstract}

Key words: galaxies: individual (NGC 3077, Garland) - galaxies: interactions - galaxies: ISM - infrared: ISM ISM: general

\section{INTRODUCTION}

It has long been known that tidal interactions can strip off the extended envelopes of atomic hydrogen $(\mathrm{HI})$ of interacting galaxies. This leads to a substantial increase of the H I cross section (i.e., areal coverage) in such systems. Given these large cross sections of atomic hydrogen, nearby interacting systems are thought to be reminiscent of damped Lyman-alpha (DLA) systems seen in absorption at high redshift.

One of the most prominent examples of a nearby interacting system is the M 81 triplet, a group of galaxies at a distance of $\sim 3.6 \mathrm{Mpc}\left(1^{\prime}=1.05 \mathrm{kpc}\right)$. Tidal $\mathrm{H}$ I features are distributed over $50 \times 100 \mathrm{kpc}^{2}$ in this system at $\mathrm{H}$ I column densities $>2 \times$ $10^{20} \mathrm{~cm}^{-2}$ and interconnect the central three galaxies M 81, M 82, and NGC 3077 (Yun et al. 1994). Early simulations suggest that the tidal system was created $3 \times 10^{8}$ years ago

\footnotetext{
${ }^{20}$ Hubble Fellow
}

and that the material in the tidal streams eastward of NGC 3077 originally belonged to NGC 3077 itself (Yun et al. 1993). The total HI mass of the tidal arm feature around NGC 3077 is $M(\mathrm{HI})=(3-5) \times 10^{8} M_{\odot}($ van der Hulst 1979; Walter \& Heithausen 1999; Walter et al. 2002a), depending on the exact integration boundaries. The $\mathrm{HI}$ emission in the tidal feature is kinematically distinct from Galactic cirrus (both in systemic velocity as well as velocity dispersion; Walter \& Heithausen 1999; Walter et al. 2002a).

However, rather than being a passive $\mathrm{H}$ I tidal feature, this region has both star formation and molecular gas, reminiscent of a dwarf galaxy. As early as 1974, Barbieri et al. (1974) detected a "fragmentary complex of almost stellar objects" in the tidal arm around NGC 3077, and subsequent optical observations revealed the presence of a blue stellar component in this region (dubbed the "Garland"; Karachentsev et al. 1985; Sharina 1991; Sakai \& Madore 2001; Mouhcine \& Ibata 2009). The presence of a young stellar population in the tidal feature has recently been 
unambigiously established by Weisz et al. (2008) using deep Hubble Space Telescope (HST) Advanced Camera for Surveys (ACS) observations.

Molecular gas has been detected and mapped in this region through observations of carbon monoxide $(\mathrm{CO}$; Walter \& Heithausen 1999; Heithausen \& Walter 2000). The total star formation rate (SFR) in the tidal feature is $2.3 \times$ $10^{-3} M_{\odot} \mathrm{yr}^{-1}$ distributed over many square-kiloparsec, comparable to what is seen in other low-mass dwarf galaxies in the M 81 group of galaxies (Walter et al. 2006). Recent optical spectroscopy of these H II regions indicated that their metallicity is much higher than expected based on the total blue magnitude of the stellar system in the tidal arm, possibly reaching a value as high as found in NGC 3077 itself, i.e., close to the Galactic one (Croxall et al. 2009). Here, we report the detection of substantial amounts of cold dust on kpc scales in the tidal feature around NGC 3077 based on Herschel SPIRE observations.

\section{OBSERVATIONS}

NGC 3077 was observed with the SPIRE instrument on Herschel (Pilbratt et al. 2010) in "scan map" mode as part of the Open Time Key Project KINGFISH (PI: R. Kennicutt) on 2010 March 28. The galaxy and its surroundings were homogeneously covered using 11 arcmin scan legs (AOR length: $0.65 \mathrm{hr}$ ). Data were reduced using the standard calibration products and algorithms available in the Herschel Interactive Processing Environment (HIPE), version 2.0.0. The determination of the background in NGC 3077 is complicated by the extended H I filaments around the region of interest. The background was defined away from the $\mathrm{H}$ i tidal tails (here defined as column densities $>2 \times 10^{20} \mathrm{~cm}^{-2}$, including NGC 3077 itself), to avoid subtracting real dust emission that may be coincident with $\mathrm{H}_{\mathrm{I}}$ features. The SPIRE data were calibrated in units of Jy beam ${ }^{-1}$, which were converted to $\mathrm{MJy} \mathrm{sr}^{-1}$ by assuming beam sizes of 501, 944, and 1924 square arcseconds (FWHM: 18", 25", and $37^{\prime \prime}$ ) for the 250, 350, and $500 \mu \mathrm{m}$ bands, respectively. For the quantitative analysis we convolved all data to the SPIRE $500 \mu \mathrm{m}$ resolution using the kernels described by Gordon et al. (2008) and updated by those authors for Herschel. We have also applied calibration correction factors of $(1.02,1.05,0.94)$ at $(250,350,500) \mu \mathrm{m}$ to the values as given in the observers manual. We selected 44 background apertures (radius: $1^{\prime}$ ) away from NGC 3077 and the tidal Hi arms. The respective flux histograms of these apertures at 250, 350, and $500 \mu \mathrm{m}$ are centered on zero, as expected given the background subtraction procedure, and have $1 \sigma$ widths of $0.33,0.16$, and $0.086 \mathrm{MJy} \mathrm{sr}^{-1}$ at 250,350 , and $500 \mu \mathrm{m}$ which we adopt as the uncertainty in the tidal feature aperture measurements (we do not include the $15 \%$ SPIRE calibration uncertainty which will affect all three bands in a similar way).

\section{RESULTS}

\subsection{Dust in the Tidal H I Arm}

Figure 1 presents a multi-wavelength view of the tidal features around NGC 3077. The top left panel shows the SPIRE map at $250 \mu \mathrm{m}$ (convolved to the $500 \mu \mathrm{m}$ beam). The brightest source in the field is NGC 3077 itself, but significant emission is detected east of the main body of the galaxy. The nuclear starburst in NGC 3077 is distributed over scales of $\sim 10^{\prime \prime}$ (Martin 1997, Ott et al. 2003, Walter et al. 2006), which is unresolved at SPIRE's resolution. The extended dust emission toward the east is spatially coincident with H I column densities $\geqslant 10^{21} \mathrm{~cm}^{-2}$ in the tidal feature (Figure 1, top right; $\mathrm{H}$ I data are taken from THINGS, "The H I Nearby Galaxy Survey"; Walter et al. 2008). The apparent correlation of H I emission with dust seen in the SPIRE data (in particular toward high H I column densities) implies that the dust emission is indeed physically coincident with the tidal arm and does not arise from Galactic cirrus which is observed toward the direction of the M 81 group (e.g., Davies et al. 2010; Sollima et al. 2010).

\subsection{Selection of Apertures}

To quantify the dust properties, we have selected 25 apertures of radius $1^{\prime}(\sim 1 \mathrm{kpc})$ each from visual inspection of the SPIRE $250 \mu \mathrm{m}$ map. The locations (and numbers) of the apertures are shown in the bottom right panel of Figure 1 (green circles) and the positions are tabulated in Table 1 . The red circle indicates the aperture (no. 1) centered on NGC 3077. For each aperture, we have derived the flux in the SPIRE bands (Table 1). The error in this measurement is derived from the dispersion of 44 background apertures of the same size that are located away from the $\mathrm{H}$ I tidal arms (at column densities $<2 \times 10^{20} \mathrm{~cm}^{-2}$ ). This dispersion is mainly driven by unresolved background sources at high redshift. The $\mathrm{H}$ i column densities (at $40^{\prime \prime}$ resolution) for each aperture are also given in Table 1 -all column densities are $>4 M_{\odot} \mathrm{pc}^{-2}\left(5 \times 10^{20} \mathrm{~cm}^{-2}\right)$. Significant dust emission (here defined as a $>3 \sigma$ detection at $250 \mu \mathrm{m}$ ) is detected in eight apertures, i.e., an area of $\sim 30 \mathrm{kpc}^{2}$. These apertures are labeled in blue in Figure 1 (bottom right) and marked with a boldface font in Table 1. Averaging the other apertures also yields a statistical detection of dust emission.

\subsection{SPIRE Dust SED}

Figure 2 shows the SPIRE measurements for NGC 3077 (aperture 1, red curve) and the sum of all detected tidal regions (green curve, boldface aperture numbers in Table 1). A comparison of the two spectral energy distributions (SEDs) directly shows that the $250 \mu \mathrm{m} / 500 \mu \mathrm{m}$ ratio is significantly lower in the tidal feature compared to the main body of NGC 3077, reflecting a lower temperature in the tidal region. Given the lower temperature in the tidal arm and the comparable total fluxes in the SPIRE bands this immediately implies that the dust mass in the tidal feature is larger than in NGC 3077 itself (see more detailed discussion in Section 3.6).

We have attempted to use Spitzer MIPS $160 \mu \mathrm{m}$ imaging to further constrain the SED toward shorter wavelengths. The main body of NGC 3077 is very bright, and we plot the corresponding flux density in Figure 2. Because of coverage and extended cirrus emission, the background of the MIPS $160 \mu \mathrm{m}$ is however badly behaved at the flux levels of interest, leading to significant error bars in the flux determination. We nevertheless derived a $160 \mu \mathrm{m}$ flux density for the brightest aperture (no. 10, the error bar is dominated by the background uncertainties)- the complete SED for this aperture is also plotted in Figure 2 for comparison.

\subsection{Dust Temperature}

We have used a blackbody fit and the Li \& Draine (2001) dust emissivity (equivalent to a modified blackbody with $\beta=2$ ) to derive temperatures and dust masses. The temperature for NGC 3077 (aperture 1) is $30.6 \pm 2 \mathrm{~K}$ and is in agreement with high temperatures expected for starbursts, and the central 


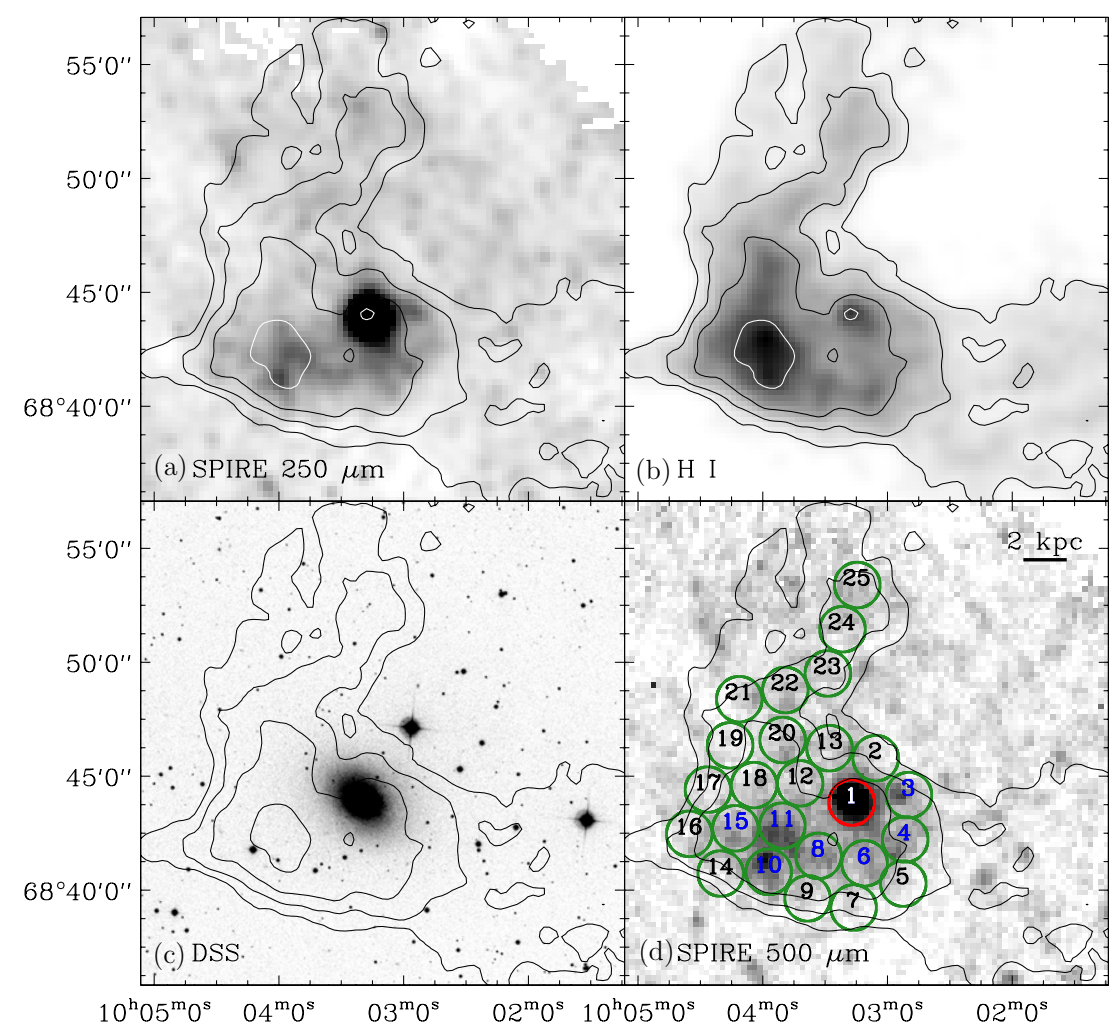

Figure 1. Multi-wavelength view of NGC 3077 and the surrounding tidal H I system (north is top, east is left). Top left: SPIRE $250 \mu \mathrm{m}$ image of NGC 3077 and its surrounding. The contours are the H I intensities shown in the top right panel. Top right: distribution of the tidal H I complex around NGC 3077 at $40^{\prime \prime}$ resolution (gray scale). Contours are shown at $\mathrm{H}$ I columns of 2, 5, 10, and $20 \times 10^{20} \mathrm{~cm}^{-2}$ at that resolution (from Walter et al. 2008). Bottom left: DSS image of NGC 3077 and its surroundings with the H I contours superimposed. Bottom right: SPIRE $500 \mu \mathrm{m}$ image, with H I contours and apertures superimposed. Aperture 1 is centered on NGC 3077 itself.

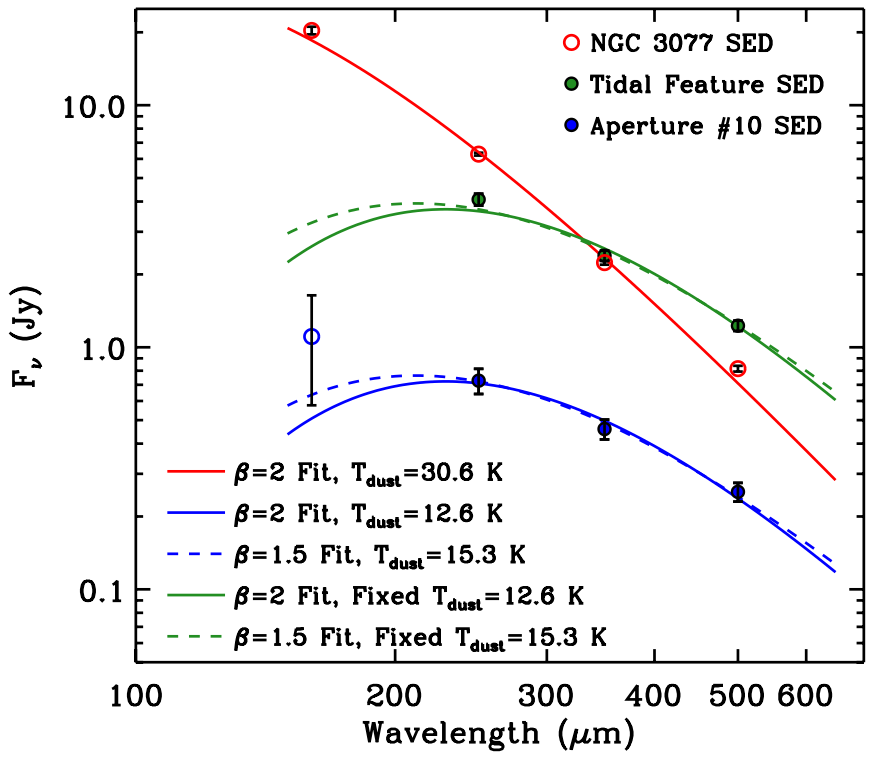

Figure 2. SPIRE dust SEDs for NGC 3077 (aperture 1, red color), the entire tidal filament (summing up apertures that contain significant emission, green), and the brightest aperture (no. 10, blue). The solid lines correspond to fits assuming $\beta=2$, the dashed curves indicate $\beta=1.5$ (fitted temperatures are given in the Figure). The SPIRE calibration uncertainties $(\sim 15 \%)$ are not included in the error bars.

temperatures derived for a sub-sample of the KINGFISH galaxies presented by Engelbracht et al. (2010) of $T_{\text {center }}=25.7 \pm$ $1 \mathrm{~K}$. The temperature in the tidal feature is much lower. Based on the SPIRE data only, we derive an average temperature of
$T=12.0 \pm 1 \mathrm{~K}$. In the brightest aperture of the tidal feature (no. 10) the addition of the $160 \mu \mathrm{m}$ flux estimate increases the temperature slightly $(12.6 \mathrm{~K})$, but is consistent with the "SPIRE-only" value. Changing $\beta$ to a value of 1.5 increases the temperature by $\sim 3 \mathrm{~K}$ (see dashed SED fits in Figure 2). In the following, we will adopt $\beta=2$ and $T=12.6 \pm 2$ for the tidal feature apertures but note that the temperature (and the corresponding masses) depends on the exact choice of dust model.

\subsection{Radiation Field}

Finding low dust temperatures may not be unexpected as the intensity of the radiation field in the tidal arm is presumably low: the total star formation in the entire tidal feature is only $2.3 \times 10^{-3} M_{\odot} \mathrm{yr}^{-1}$ (based on $\mathrm{H} \alpha$ observations; Walter et al. 2006). This SFR is consistent with the rate determined in Weisz et al. (2008) through stellar population studies, and GALEX measurements that cover regions 10 and 11 , implying that the SFR in the tidal feature was roughly constant over the recent past (few hundred million years). For those apertures that include $\mathrm{H}$ II regions we sum up their $\mathrm{H} \alpha$ luminosities using the values given in Walter et al. (2006) and give the SFR surface densities (averaged over the size of our apertures) in Table 1. We plot the $250 \mu \mathrm{m} / 500 \mu \mathrm{m}$ ratios for these aperatures as a function of the $\mathrm{H} \alpha$ luminosity surface density in Figure 3. SFRs can be estimated using $\operatorname{SFR}\left[M_{\odot} \mathrm{yr}^{-1}\right]=$ $7.9 \times 10^{-42} \mathrm{~L}(\mathrm{H} \alpha)\left[\mathrm{erg} \mathrm{s}^{-1}\right.$ ] (Kennicutt 1998, see top $x$-axis in Figure 3; choosing a Kroupa IMF would decrease the SFRs by $\sim 30 \%$ ) and the brightest region outside of NGC 3077 (aperture 10) accounts for $\sim 50 \%$ of the total SFR in the tidal complex 
Table 1

Aperture Measurements

\begin{tabular}{|c|c|c|c|c|c|c|c|c|}
\hline No. $^{a}$ & $\begin{array}{c}\text { R.A. } \\
\text { J2000.0 }\end{array}$ & $\begin{array}{c}\text { Decl. } \\
\text { J2000.0 }\end{array}$ & $\begin{array}{l}\mathrm{F}(250 \mu \mathrm{m})^{\mathrm{b}} \\
\left(\mathrm{MJy} \mathrm{sr}^{-1}\right)\end{array}$ & $\begin{array}{l}\mathrm{F}(350 \mu \mathrm{m})^{\mathrm{b}} \\
\left(\mathrm{MJy} \mathrm{sr}^{-1}\right)\end{array}$ & $\begin{array}{l}\mathrm{F}(500 \mu \mathrm{m})^{\mathrm{b}} \\
\left(\mathrm{MJy} \mathrm{sr}^{-1}\right)\end{array}$ & $\begin{array}{c}\Sigma_{\mathrm{dust}^{\mathrm{c}}} \\
\left(M_{\odot} \mathrm{pc}^{-2}\right)\end{array}$ & $\begin{array}{c}\Sigma_{\mathrm{H}_{\mathrm{I}}}{ }^{\mathrm{d}} \\
\left(M_{\odot} \mathrm{pc}^{-2}\right)\end{array}$ & $\frac{\Sigma_{\mathrm{SFR}}^{\mathrm{e}}}{\left(10^{-4} M_{\odot} \mathrm{yr}^{-1} \mathrm{kpc}^{-2}\right)}$ \\
\hline 1 & 100317.18 & +684353.0 & 23.61 & 8.41 & 3.07 & $0.054 \pm 0.005$ & 12.42 & 220 \\
\hline 2 & 100305.57 & +684549.4 & 0.73 & 0.34 & 0.15 & $\ldots$ & 2.95 & $\cdots$ \\
\hline 3 & 100249.53 & +684412.2 & 1.54 & 0.91 & 0.48 & $0.053 \pm 0.015$ & 4.88 & $\ldots$ \\
\hline 4 & 100251.35 & +684215.7 & 1.71 & 0.99 & 0.53 & $0.058 \pm 0.016$ & 7.04 & $\cdots$ \\
\hline 5 & 100252.28 & +684019.3 & 0.02 & 0.11 & 0.09 & $\ldots$ & 6.04 & $\ldots$ \\
\hline 6 & 100310.96 & +684112.8 & 2.63 & 1.32 & 0.58 & $0.075 \pm 0.020$ & 10.18 & $\cdots$ \\
\hline 7 & 100316.30 & +683916.3 & -0.10 & 0.05 & 0.05 & $\ldots$ & 5.63 & $\cdots$ \\
\hline 8 & 100333.21 & +684132.2 & 2.53 & 1.35 & 0.65 & $0.078 \pm 0.021$ & 10.69 & 0.65 \\
\hline 9 & 100338.53 & +683940.5 & 0.29 & 0.31 & 0.17 & $\ldots$ & 7.69 & $\ldots$ \\
\hline 10 & 100357.30 & +684050.1 & 2.74 & 1.73 & 0.95 & $0.101 \pm 0.027$ & 14.78 & 4.00 \\
\hline 11 & 100350.72 & +684251.7 & 2.65 & 1.68 & 0.91 & $0.097 \pm 0.026$ & 16.23 & 1.23 \\
\hline 12 & 100342.18 & +684443.2 & 0.76 & 0.57 & 0.34 & $\ldots$ & 8.16 & $\ldots$ \\
\hline 13 & 100327.96 & +684615.9 & 0.84 & 0.51 & 0.31 & $\cdots$ & 3.30 & $\cdots$ \\
\hline 14 & 100420.44 & +684045.2 & 0.63 & 0.41 & 0.21 & $\ldots$ & 6.39 & $\cdots$ \\
\hline 15 & 100413.43 & +684244.4 & 1.54 & 1.02 & 0.52 & $0.057 \pm 0.016$ & 15.62 & 0.29 \\
\hline 16 & 100435.72 & +684229.4 & 0.59 & 0.41 & 0.21 & $\ldots$ & 6.30 & 1.09 \\
\hline 17 & 100426.79 & +684426.1 & 0.19 & 0.16 & 0.10 & $\cdots$ & 5.23 & $\cdots$ \\
\hline 18 & 100404.49 & +684438.4 & 0.63 & 0.42 & 0.26 & $\ldots$ & 12.60 & $\ldots$ \\
\hline 19 & 100416.16 & +684622.8 & -0.23 & -0.07 & 0.01 & $\cdots$ & 6.49 & $\cdots$ \\
\hline 20 & 100350.70 & +684637.3 & 0.54 & 0.43 & 0.31 & $\cdots$ & 8.93 & $\cdots$ \\
\hline 21 & 100411.77 & +684824.2 & 0.04 & 0.11 & 0.13 & $\ldots$ & 4.71 & $\ldots$ \\
\hline 22 & 100349.41 & +684848.8 & 0.89 & 0.51 & 0.29 & $\cdots$ & 5.94 & $\ldots$ \\
\hline 23 & 100328.82 & +684932.7 & 0.72 & 0.39 & 0.19 & $\ldots$ & 4.58 & $\ldots$ \\
\hline 24 & 100321.66 & +685129.2 & 0.78 & 0.42 & 0.23 & $\ldots$ & 5.35 & $\ldots$ \\
\hline 25 & 100314.47 & +685325.6 & 0.78 & 0.38 & 0.24 & $\cdots$ & 4.36 & $\cdots$ \\
\hline
\end{tabular}

Notes.

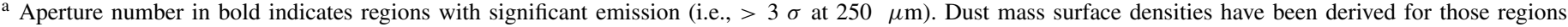
assuming a temperature of $T=12.6 \mathrm{~K}$ (only exception: aperture 1 [NGC 3077, $T=30.6 \mathrm{~K}]$ ) and $\beta=2$ (see Section 3.4 ).

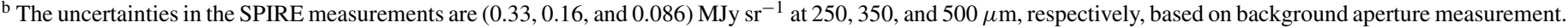
These uncertainties do not include the SPIRE calibration uncertainties of $\sim 15 \%$.

${ }^{c}$ The uncertainty in dust mass surface densities have been derived using different temperatures $( \pm 1 \mathrm{~K})$ as the fitting errors give unrealistically small numbers.

${ }^{\mathrm{d}} \mathrm{H}_{\mathrm{I}}$ surface density uncertainties are typically $<10 \%$.

e SFR surface densities are derived from the values given in Walter et al. (2006; tidal arm) and Kennicutt et al. (2008; NGC 3077).

$\left(1.3 \times 10^{-3} M_{\odot} \mathrm{yr}^{-1}\right.$ or a SFR surface density for this aperture of $4 \times 10^{-4} M_{\odot} \mathrm{yr}^{-1} \mathrm{kpc}^{-2}$ ). For reference, single massive stars create $\mathrm{H}_{\mathrm{II}}$ regions with $\mathrm{H} \alpha$ luminosities of $L_{\mathrm{H} \alpha, \mathrm{O} 7} \approx$ $5 \times 10^{36} \mathrm{erg} \mathrm{s}^{-1}$ and $L_{\mathrm{H} \alpha, \mathrm{O} 5} \approx 5 \times 10^{37} \mathrm{erg} \mathrm{s}^{-1}$ (e.g., Devereux et al. 1997), i.e., the total $\mathrm{H} \alpha$ flux in the tidal feature can in principle be created by a few massive stars. Figure 3 shows that the dust temperature does not change as a function of the SFR surface density over two orders of magnitude of the latter. This suggests that the currently observed star formation does not provide the main heating source for the dust on kpc scales and we speculate that other mechanisms may be responsible for the dust heating.

Assuming $G_{0} \sim 4 \times 10^{-3}$ for the intergalactic radiation field (Sternberg et al. 2002, note that $G_{0}=1$ for the Galactic interstellar radiation field), that the temperature of dust goes as $T \sim G_{0}^{1 /(4+\beta)}$ and $\beta=2$, and scaling from the results by Li \& Draine (2001) for the Galactic interstellar radiation field (16 K for a silicate grain, $20 \mathrm{~K}$ for a graphite grain, Figure 3 in Li \& Draine 2001), we estimate that the dust in the tidal feature should have a temperature of $6-8 \mathrm{~K}$ in the intergalactic field. This suggests that other heating sources (the radiation field of NGC 3077, an older stellar population in the feature as seen in the HST imaging by Weisz et al. 2008 and/or shocks) must be partly responsible for heating the dust to our derived temperature of $12 \pm 2 \mathrm{~K}$. The $250 \mu \mathrm{m} / 500 \mu \mathrm{m}$ ratio does not

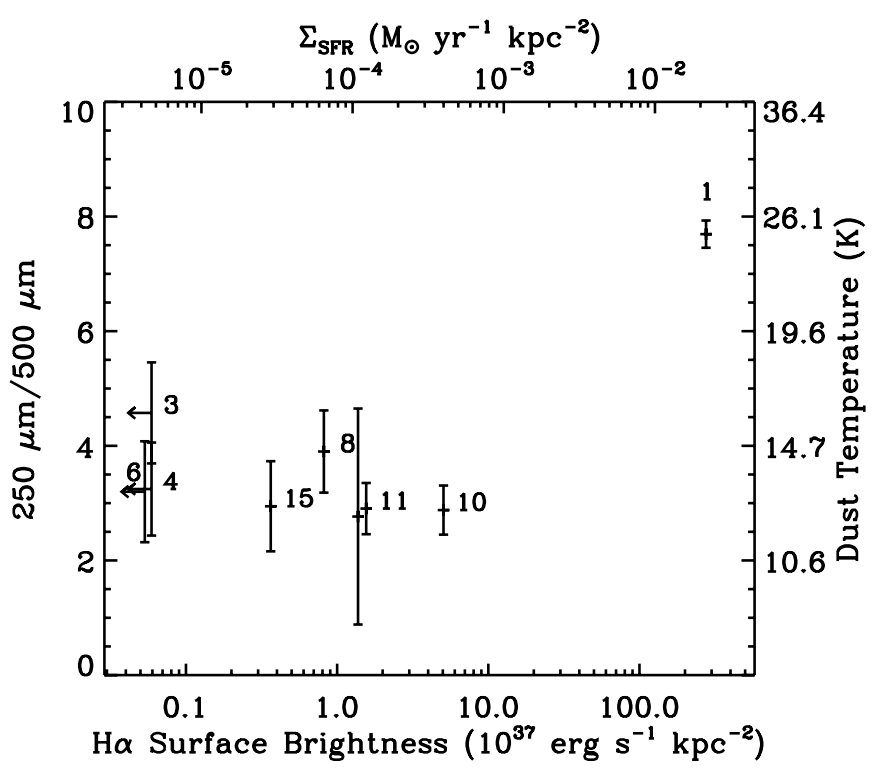

Figure 3. $250 \mu \mathrm{m} / 500 \mu \mathrm{m}$ ratio in the tidal feature as a function of $\mathrm{H} \alpha$ luminosity in a given aperture (numbers indicate aperture numbers). The temperatures given on the right $y$-axis are based on this $250 \mu \mathrm{m} / 500 \mu \mathrm{m}$ ratio and assume a blackbody with $\beta=2$. 
change as a function of projected distance to NGC 3077 within our uncertainties but we note that the measurements only span a small range in distances.

\subsection{Dust Masses and Dust/Gas Ratios}

From the SED fits discussed above we derive dust mass surface densities for each of our apertures (Table 1) with an average value of $7.4 \times 10^{-2} M_{\odot} \mathrm{pc}^{-2}(\beta=2, T=12.6 \mathrm{~K})$. We derive a total dust mass in the tidal feature (excluding NGC 3077 and upper limits) of $\sim(1.8 \pm 0.9) \times 10^{6} M_{\odot}$ (the large error bar includes uncertainties in dust properties, see below). Our simple dust mass estimate is consistent with results from more detailed modeling using updated Draine \& Li (2007) models (G. Aniano et al. 2011, in preparation). The dust mass of NGC 3077 is only $1.9 \times 10^{5} M_{\odot}(\beta=2$ and $T=30.6 \mathrm{~K})$, in reasonable agreement with the mass estimates by Price \& Gullixson (1989) of the absorbing central dust clouds based on NIR observations (they report a mass of $\sim 10^{5} M_{\odot}$ ). Both mass estimates depend on the choice of $\beta$ and the temperature. For example, if we chose $\beta=1.5$ for the tidal feature, the dust mass would go down by a factor of $\sim 2$ (as the temperature would increase by $\sim 3 \mathrm{~K}$; Section 3.4). Likewise, lowering the dust temperature in NGC 3077 by $5 \mathrm{~K}$ would increase its dust mass by a factor of $\sim 2$. Given these large systematic uncertainties involved we assign a conservative error of $50 \%$ to the dust mass of the tidal feature.

For the average Hi surface mass densities of the apertures where dust emission was significantly detected, we derive an average value of $11.3 M_{\odot} \mathrm{pc}^{-2}$ (Table 1), i.e., more than two orders of magnitude larger than the dust surface densities. The average dust-to-H I gas ratio is $(6.5 \pm 3) \times 10^{-3}$, and the ratio does not vary within the errors between apertures and is not a function of H I surface density (the corresponding value for NGC 3077 (aperture 1) is 0.0043). Within the uncertainties this value is close to the average dust-to-gas ratio of $M_{\text {dust }} /$ $M_{\mathrm{H}} \approx 0.005$ derived by Draine et al. (2007) for 12 spiral galaxies of the SINGS sample for which Spitzer and SCUBA measurements were available. As noted by Draine et al. (2007), an $M_{\text {dust }} / M_{\mathrm{H} \text { I }}$ ratio between 0.003 and 0.01 is consistent with galactic metallicities between 0.3 and 1 times solar, if a similar fraction of heavy elements is in the form of dust as in the Milky Way. Our derived dust-to-gas ratio thus indicates that the tidal arm is substantially chemically enriched, in agreement with the H II region metallicities derived by Croxall et al. (2009). For comparison, the $M_{\text {dust }} / M_{\mathrm{HI}}$ ratio for metal-poor dwarf irregular galaxies in the (more extended) M 81 group of galaxies is up to one order of magnitude lower than found here (Walter et al. 2007).

So far, we have only considered the $\mathrm{H}$ I gas. Molecular gas has been mapped in the $\mathrm{CO}(1-0)$ transition in the tidal feature over a restricted area (Walter \& Heithausen 1999; Heithausen \& Walter 2000; Walter et al. 2006). The two main molecular complexes (region numbers 1 and 2 in Heithausen \& Walter 2000) are spatially coincident with aperture numbers 10 and 11 in this study. The implied molecular gas masses are dependent on the choice of the $\mathrm{H}_{2}$-to-CO conversion factor $\mathrm{X}_{\mathrm{CO}}$. Heithausen \& Walter (2000) used a conversion factor of $\mathrm{X}_{\mathrm{CO}}=8 \times 10^{20} \mathrm{~cm}^{-2}$ $\left(\mathrm{K} \mathrm{km} \mathrm{s}^{-1}\right)^{-1}$, a factor of $\sim 4$ larger than the Galactic conversion factor. At the time, their choice was driven by the assumption that the tidal region was metal-poor. Given the abundance of dust and dust-to-gas ratios similar to nearby spiral galaxies, as well as the $\mathrm{H}$ II region metallicity measurements (indicating metallicities as high as Galactic; Croxall et al. 2009), it now seems to be more appropriate to use the Galactic conversion factor in this tidal feature. This implies that the masses given in Heithausen $\&$ Walter (2000) should be divided by a factor of four. We thus adopt molecular gas masses of $\sim 1 \times 10^{6} M_{\odot}$ and $\sim 4 \times 10^{6} M_{\odot}$ for our apertures 10 and 11, respectively (comparable to the $\mathrm{H}_{2}$ mass present in the center of NGC 3077; Meier et al. 2001; Walter et al. 2002a). Averaged over our $r=1 \mathrm{kpc}$ sized apertures this corresponds to a molecular surface density between 1.1 and $0.3 M_{\odot} \mathrm{pc}^{-2}$ (i.e., the interstellar medium, ISM, in the tidal feature is dominated by the atomic gas phase even in the CO-brightest regions.

\section{CONCLUDING REMARKS}

The detection of a significant amount of dust $(\sim(1.8 \pm 0.9)$ $\times 10^{6} M_{\odot}$ ) in the tidal feature near NGC 3077, distributed over $30 \mathrm{kpc}^{2}$, which is significantly larger than the dust mass present in the parent galaxy (irrespective of the choice of $\beta$ and reasonable temperatures), raises questions on the origin of the enriched material. The ongoing star formation could potentially enrich the medium in the tidal feature. If we assumed a constant SFR since the creation of the feature $\left(3 \times 10^{8}\right.$ yr ago; Yun et al. 1994), we estimate a total mass of newly formed stars of $\sim 7 \times 10^{5} M_{\odot}$, i.e., less than the total amount of dust that is present. This implies that the current rate of star formation activity cannot have created the present dust. Also, the chemical enrichment due to the $\mathrm{H}$ II regions in the tidal arm is expected to lead to a metallicity of only $Z \sim 0.002$ Solar (Walter et al. 2006) over the last $3 \times 10^{8} \mathrm{yr}$, i.e., much lower than what is measured by Croxall et al. (2009), and implied by our dust-to-gas ratio. We conclude that the tidal arm material was pre-enriched and likely belonged to NGC 3077 (which has a metallicity similar to the tidal H II regions; Croxall et al. 2009) before the interaction.

Our findings imply that interactions between galaxies can efficiently remove heavy elements, dust, and molecules from a galaxy. In the case discussed here, significantly more dust mass is found in the tidal arm than in the parent galaxy NGC 3077 (the same holds true for the atomic and molecular gas). Interactions thus appear to have the potential to alter the chemical evolution of a galaxy dramatically and to expel an ISM that is enriched by heavy elements. As interactions have been more frequent at larger look-back times it is conceivable that this mechanism can (in addition to outflows; e.g., Steidel et al. 2010) effectively enrich the intergalactic medium (see also Roussel et al. 2010; Walter et al. 2002b). The tidal system discussed here would be classified as a DLA absorber with a cross section of roughly $30 \mathrm{kpc}^{2}$ (e.g., Wolfe et al. 2005; Zwaan et al. 2008).

F.W. acknowledges the hospitality of the Aspen Center for Physics. Herschel is an ESA space observatory with science instruments provided by European-led Principal Investigator consortia and with important participation from NASA.

\section{REFERENCES}

Barbieri, C., Bertola, F., \& di Tullio, G. 1974, A\&A, 35, 463

Croxall, K. V., van Zee, L., Lee, H., Skillman, E. D., Lee, J. C., Côté, S., Kennicutt, R. C., \& Miller, B. W. 2009, ApJ, 705, 723

Davies, J. I., et al. 2010, MNRAS, 409, 102

Devereux, N., Duric, N., \& Scowen, P. A. 1997, AJ, 113, 236

Draine, B. T., \& Li, A. 2007, ApJ, 657, 810

Draine, B. T., et al. 2007, ApJ, 663, 866

Engelbracht, C. W., et al. 2010, A\&A, 518, L56

Gordon, K. D., Engelbracht, C. W., Rieke, G. H., Misselt, K. A., Smith, J.-D. T., \& Kennicutt, R. C., Jr. 2008, ApJ, 682, 336

Heithausen, A., \& Walter, F. 2000, A\&A, 361, 500 
Karachentsev, I. D., Karachentseva, V. E., \& Börngen, F. 1985, MNRAS, 217, 731

Kennicutt, R. C., Jr. 1998, ARA\&A, 36, 189

Kennicutt, R. C., Jr., Lee, J. C., Funes, S. J., José, G., Sakai, S., \& Akiyama, S. 2008, ApJS, 178, 247

Li, A., \& Draine, B. T. 2001, ApJ, 554, 778

Martin, C. L. 1997, ApJ, 491, 561

Meier, D. S., Turner, J. L., \& Beck, S. C. 2001, AJ, 122, 1770

Mouhcine, M., \& Ibata, R. 2009, MNRAS, 399, 737

Ott, J., Martin, C. L., \& Walter, F. 2003, ApJ, 594, 776

Pilbratt, G. L., et al. 2010, A\&A, 518, L1

Price, J. S., \& Gullixson, C. A. 1989, ApJ, 337, 658

Roussel, H., et al. 2010, A\&A, 518, L66

Sakai, S., \& Madore, B. F. 2001, ApJ, 555, 280

Sharina, M. E. 1991, Sov. Astron. Lett., 17, 383

Sollima, A., Gil de Paz, A., Martinez-Delgado, D., Gabany, R. J., GallegoLaborda, J. J., \& Hallas, T. 2010, A\&A, 516, A83

Steidel, C. C., Erb, D. K., Shapley, A. E., Pettini, M., Reddy, N., Bogosavljević, M., Rudie, G. C., \& Rakic, O. 2010, ApJ, 717, 289
Sternberg, A., McKee, C. F., \& Wolfire, M. G. 2002, ApJS, 143, 419

van der Hulst, J. M. 1979, A\&A, 75, 97

Walter, F., Brinks, E., de Blok, W. J. G., Bigiel, F., Kennicutt, R. C., Thornley, M. D., \& Leroy, A. 2008, AJ, 136, 2563

Walter, F., \& Heithausen, A. 1999, ApJ, 519, L69

Walter, F., Martin, C. L., \& Ott, J. 2006, AJ, 132, 2289

Walter, F., Weiss, A., Martin, C., \& Scoville, N. 2002a, AJ, 123, 225

Walter, F., Weiss, A., \& Scoville, N. 2002b, ApJ, 580, L21

Walter, F., et al. 2007, ApJ, 661, 102

Weisz, D. R., Skillman, E. D., Cannon, J. M., Dolphin, A. E., Kennicutt, R. C., Jr., Lee, J., \& Walter, F. 2008, ApJ, 689, 160

Wolfe, A. M., Gawiser, E., \& Prochaska, J. X. 2005, ARA\&A, 43, 861

Yun, M. S., Ho, P. T. P., Brouillet, N., \& Lo, K. Y. 1993, in Evolution of Galaxies and their Environment, ed. D. Hollenbeck et al. (NASA Conf. Pub. no. 3190; Washington, DC: NASA), 253

Yun, M. S., Ho, P. T. P., \& Lo, K. Y. 1994, Nature, 372, 530

Zwaan, M., Walter, F., Ryan-Weber, E., Brinks, E., de Blok, W. J. G., \& Kennicutt, R. C. 2008, AJ, 136, 2886 